\title{
Linking Environmental Science with Geochemistry
}

\author{
Wei Li ${ }^{1}$ (I) Jianbo $\mathrm{Shi}^{2} \cdot$ Lei Huang $^{3} \cdot$ Xiaosan Luo $^{4} \cdot$ Pei Lei $^{3}$
}

Received: 6 December 2020 / Accepted: 14 December 2020 / Published online: 4 January 2021

(C) The Author(s), under exclusive licence to Springer Science+Business Media, LLC part of Springer Nature 2021

This is a special issue dedicated to interdisciplinary research between environmental science and geochemistry. Many of the studies included in this issue were presented in the Session of Environmental Science of the 6th Young Scientist Forum of Earth Science (http://www.qndxlt.com/) that took place in Xining, China in October, 2019. Many excellent young environmental scientists and geochemists presented their most updated research progress in this session, making it a convergence of environmental science and geochemistry, which eventually inspired the organization of this special issue. This special issue also hosts studies by other researchers working at the interface of environmental science and geochemistry.

In particular, this special issue is divided into two parts. The first part is focused on laboratory-based studies on the environmental processes of contaminants in mimic environmental conditions. These contaminants cover mainly on toxic metals such as $\mathrm{As}, \mathrm{Se}, \mathrm{Cd}, \mathrm{Cr}, \mathrm{U}$ to examine their sorption and co-precipitation on environmental mineral surfaces or spatial distributions in environmental samples. The second part focused on field scale studies to understand natural distributions and bioavailability of inorganic (e.g., $\mathrm{N}$, $\mathrm{P}, \mathrm{Cd}, \mathrm{Hg}$ ) and organic contaminants (e.g., PAHs) in natural settings. The field sites cover water bodies spanning from Guanting Reservoir to Taihu Lake to Pearl River Delta, and terrestrial lands spanning from paddy soils to Karst highland located in Southwest China. We are thrilled to see these excellent researches focused different scales perfectly fused into this special issue, which largely improved our understanding of the complex and dynamic environmental system. The knowledge will undoubtedly assist in environmental mitigation and regulation and will also provide scientific basis for policy makers in choosing strategies for environmental protection.

The Guest Editors would like to thank Dr. Erin Bennett, the Editor-in-Chief and Dr. Huan Zhong, the Executive Associate Editor, Dr. Guangxia Liu, the Assistant Editor of Bulletin of Environmental Contamination and Toxicology, for their help and support to this special issue. Of course, we are grateful to the authors and the reviewers for their efforts.

Wei Li

liwei_isg@nju.edu.cn

1 Key Lab of Surficial Geochemistry (Ministry of Education), School of Earth Sciences \& Engineering, Nanjing University, Nanjing 210023, China

2 State Key Lab of Environmental Chemistry \& Ecotoxicology, Research Center for Eco-Environmental Sciences, Chinese Academy of Sciences, Beijing 10085, China

3 State Key Laboratory of Pollution Control and Resources Reuse, School of the Environment, Nanjing University, Nanjing 210023, China

4 International Center for Ecology, Meteorology, and Environment, Nanjing University of Information Science \& Technology, Nanjing 210044, Jiangsu, China 